\title{
Positive response of a primary malignant pericardial mesothelioma to pemetrexed plus cisplatin followed by pemetrexed maintenance chemotherapy: A case report
}

\author{
SANG MI CHUNG，SEONG JI CHOI，MIN JUNG KIM，JUNG YOON CHOI, \\ HONG JUN KIM, SUK-YOUNG LEE and EUN JOO KANG
}

Division of Medical Oncology, Department of Internal Medicine, Korea University College of Medicine, Korea University Medical Center, Guro Hospital, Seoul 08308, Republic of Korea

Received March 30, 2015; Accepted May 5, 2016

DOI: $10.3892 / 01.2016 .4598$

\begin{abstract}
Primary malignant pericardial mesothelioma (PMPM) is a rare tumor with poor prognosis. Surgery is the treatment of choice, but numerous cases are inoperable. For the treatment of inoperable or metastatic cases, systemic chemotherapy is required. However, a standard chemotherapeutic regimen for the treatment of pericardial mesothelioma has not yet been established. Chemotherapy involving pemetrexed and cisplatin has been actively used in the treatment of pleural or peritoneal mesothelioma, and may be considered for the treatment of PMPM. The present study reports the case of a patient with PMPM with lung metastasis who demonstrated a positive response to treatment with pemetrexed and cisplatin followed by pemetrexed maintenance chemotherapy, leading to prolonged progression-free survival for 21 months.
\end{abstract}

\section{Introduction}

Primary malignant pericardial mesothelioma (PMPM) is an extremely rare tumor that develops in mesothelial cells of the pericardium (1). Malignant mesothelioma typically arises from the pleura (65-70\%) and peritoneum (30\%) (2). Those tumors arising from the pericardium account for only 1-2\% of all mesotheliomas (3). The incidence of PMPM is extremely low, with $<0.0022 \%$ of cases reported in a large-scale autopsy study (1). PMPM has been reported predominantly in men compared with women, with $2: 1$ and 3:1 ratios $(2,4)$. The disease presents with non-specific symptoms, including dyspnea or chest

Correspondence to: Dr Eun Joo Kang, Division of Medical Oncology, Department of Internal Medicine, Korea University College of Medicine, Korea University Medical Center, Guro Hospital, 148 Gurodong-Ro, Guro-Gu, Seoul 08308, Republic of Korea

E-mail:kkangju11@naver.com

Key words: malignant mesothelioma, pericardium, chemotherapy, pemetrexed, cisplatin pain $(4,5)$. Initial manifestations, including pericardial effusion or pericardial thickening, frequently result in confusion with pericarditis or heart failure and delay diagnosis $(4,6,7)$. Therefore, multi-modal imaging methods, including echocardiography, computed tomography (CT), magnetic resonance imaging (MRI) and ${ }^{18} \mathrm{~F}$-fluorodeoxyglucose-positron emission tomography, are often required for the diagnosis $(8,9)$. Tumors are located in a poorly resectable area and tend to infiltrate adjacent structures such as the great vessels, therefore, complete surgical removal is often not possible $(5,10)$. As a result, surgical resection combined with radiotherapy or chemotherapy is required in cases of localized tumors $(11,12)$. In addition, numerous patients diagnosed with PMPM have accompanying distant metastasis $(13,14)$. For such cases, systemic chemotherapy is important $(11,14)$. However, there are currently no existing standard chemotherapeutic drugs or treatment strategies, and as a result overall prognosis remains poor at $\sim 6$ months $(2,4,5,11,13)$. The present study reports the case of a patient diagnosed with PMPM with accompanying lung metastasis. The patient demonstrated a positive response to pemetrexed plus cisplatin followed by pemetrexed monotherapy, leading to prolonged progression-free survival for 21 months.

\section{Case report}

A 53-year-old woman presented to Korea University Medical Center, Guro Hospital (Seoul, South Korea) with peripheral edema, dyspnea on exertion, coughing and intermittent fever in May 2013. In February 2007, the patient had presented to the hospital with massive pericardial effusion. An extensive investigation, including pericardial biopsy, was performed to identify the cause. However, there was no evidence linking the symptoms to viruses, bacteria or malignancy. Chronic inflammation with fibrosis was observed in a pericardial biopsy. Following pericardiocentesis and treatment with diuretics, pericardial effusion resolved and did not recur. The patient had maintained good health for 6 years until the current presentation.

The patient was a non-smoker, occasionally drank alcohol and had a history of working in a small factory making electrical units. The patient had no other occupational or environmental 
A

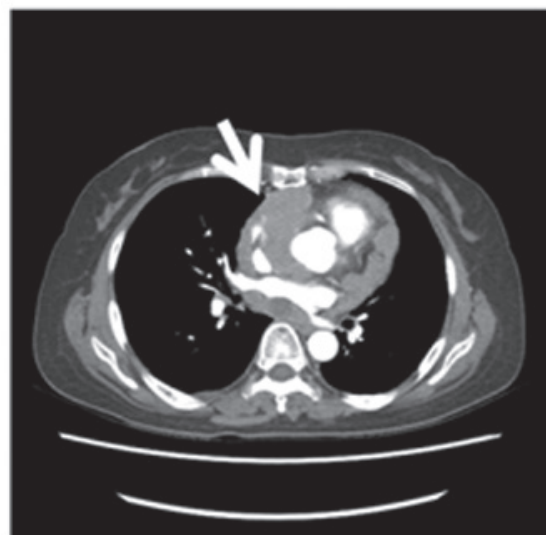

C

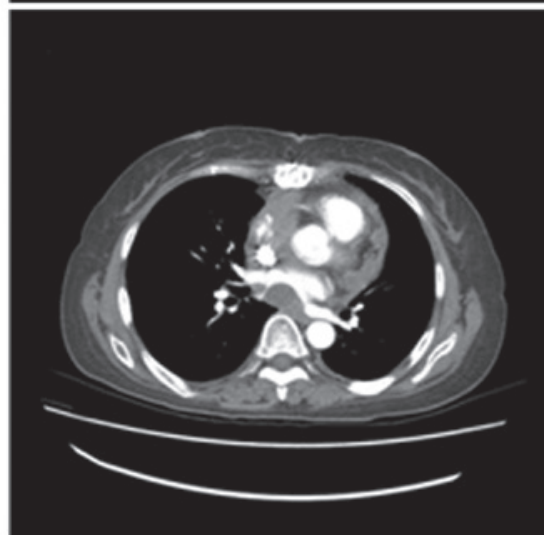

$\mathbf{E}$

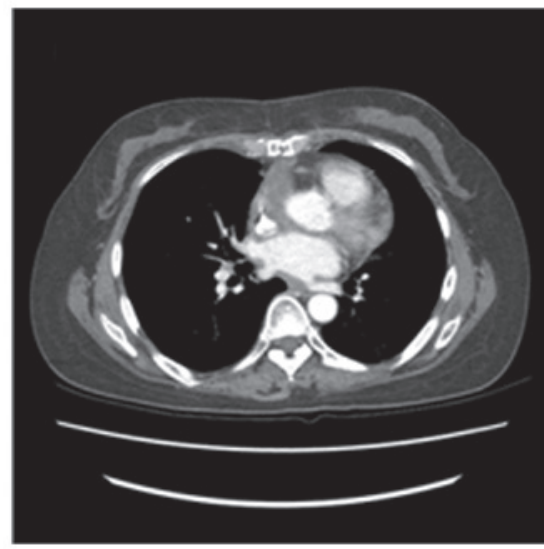

B

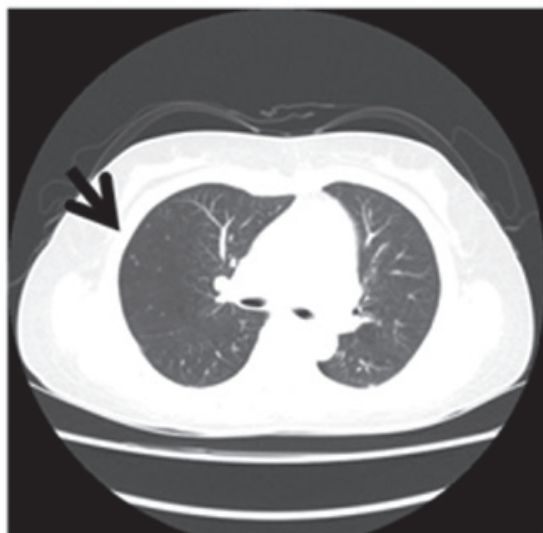

D

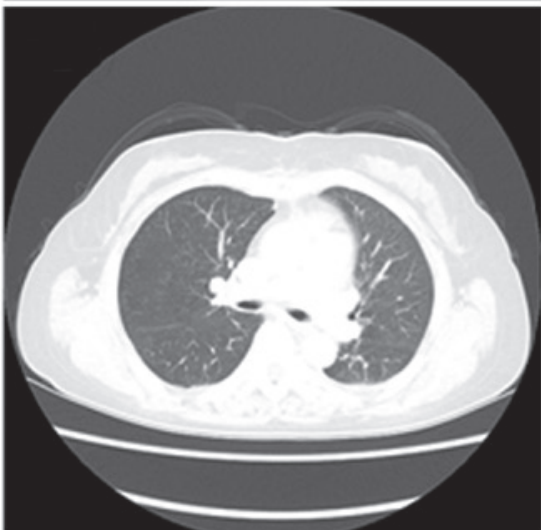

$\mathbf{F}$

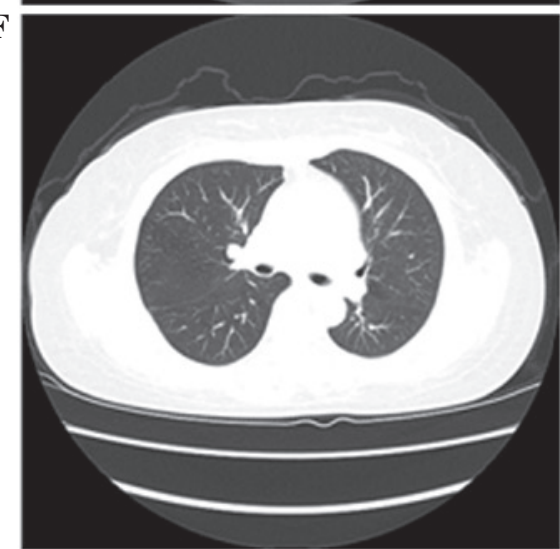

Figure 1. (A) CT scan of the chest at the time of diagnosis showing diffuse thickening of the pericardium (white arrow). (B) CT scan of the chest at the time of diagnosis showing multiple metastatic nodules in the lung (black arrow). (C and D) Regression of the pericardial thickening and a decreasing number of multiple metastatic lung nodules were exhibited following treatment with pemetrexed plus cisplatin. (E and F) Additional regression of the pericardial thickening with no change in the number of multiple lung nodules were observed following continuation of pemetrexed monotherapy. CT, computed tomography.

exposure to asbestos. Vital signs were stable upon admission to Korea University Medical Center, Guro Hospital in 2013. The patient's jugular venous pressure was elevated and she was positive for the Kussmaul's sign (15). No additional information, except for mild cardiomegaly, was revealed by chest radiography. Transthoracic echocardiography revealed a newly developed pericardial thickening and mass-like lesion encircling the ascending aorta. However, the ejection fraction was within the normal range. CT of the chest revealed diffuse pericardial thickening and pericardial masses surrounding the entire pericardium (Fig. 1A). Cardiac MRI revealed enhancement of the mass with internal necrosis. Pericardiectomy for histological confirmation and biopsy of the periaortic lymph node near the mass were performed. Upon examination for distant metastasis, multiple tiny nodules, which appeared to be metastatic, were observed in both lung fields on a chest CT (Fig. 1B). No other distant metastases were identified. Therefore, palliative chemotherapy was initiated with pemetrexed $\left(500 \mathrm{mg} / \mathrm{m}^{2}\right)$ and cisplatin $\left(75 \mathrm{mg} / \mathrm{m}^{2}\right)$ via intravenous administration in a 3 -week schedule. Following the second cycle of treatment, the pericardial thickening began to regress. The patient continued to receive chemotherapy and the tumor size and number of lung metastases reduced continuously (Fig. 1C and D). From the tenth cycle, the dose of cisplatin was reduced to $50 \%$ of the full dose $\left(35 \mathrm{~g} / \mathrm{m}^{2}\right)$ due to grade 2 peripheral neuropathy. Following the fourteenth cycle, only pemetrexed was infused every 3 weeks. The tumor size reduced further and maintained this reduced status (Fig. 1E and F).

Using a light microscope, the excised pericardial soft tissue confirmed the diagnosis of malignant pericardial mesothelioma. 

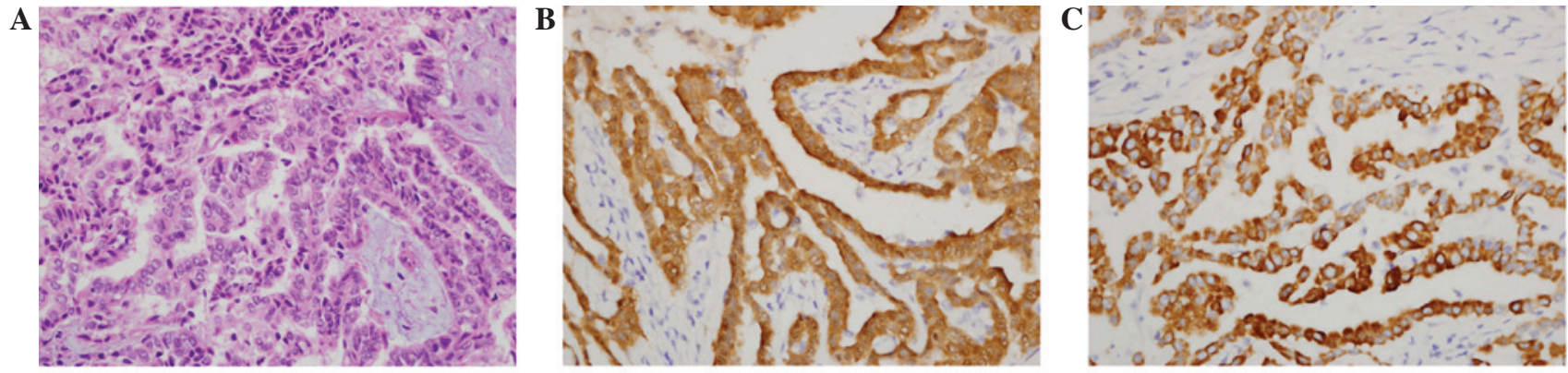

Figure 2. (A) Microscopic examination of the biopsy specimen showed proliferation of mesothelial cells with pleomorphic nuclei, consistent with epithelioid-type malignant mesothelioma (hematoxylin and eosin staining; magnification, $\mathrm{x} 400$ ). (B) Immunohistochemistry revealed positive staining for calretinin (magnification, x200). (C) Immunohistochemistry revealed positive staining for cytokeratin 5/6 (magnification, x200).

Tumor tissue was fixed in $10 \%$-formalin and embedded in paraffin. Paraffin sections were routinely processed and stained with hematoxylin and eosin. The specimens showed neoplastic cells with epithelioid and papillary patterns (Fig. 2A). Immunohistochemistry was performed using the BOND MAX system (Leica Biosystems, Newcastle, UK) with the following antibodies: Monoclonal mouse anti-human calretinin (dilution, 1:1,200; catalog no. NCL-L-CALRET-566; Leica Biosystems), monoclonal mouse anti-human cytokeratin 5/6 (dilution, 1:1,200; catalog no. M7237; Dako, Glostrup, Denmark), monoclonal mouse anti-human carcinoembryonic antigen (dilution, 1:200; catalog no. M7072; Dako), monoclonal mouse anti-human thyroid transcription factor-1 (TTF-1; dilution, 1:200; catalog no. M3675; Dako) and monoclonal mouse anti-human Wilms' Tumor-1 (WT-1; dilution, 1:100; catalog no. NCL-L-WT1-562; Leica Biosystems). Immunohistochemistry revealed that the specimens were positive for calretinin and cytokeratin $5 / 6$ (Fig. 2B and C), but negative for carcinoembryonic antigen, TTF-1 and WT-1 protein. These findings were consistent with those from the excised periaortic lymph node.

Following treatment, the patient developed intermittent grade 3 neutropenia but recovered with no other complications. The patient has been treated with pemetrexed with regular CT scans every 3 months. The patient remains in good medical condition 21 months after treatment initiation.

Informed consent was obtained from the patient for publication of this study, and the study was approved by the Ethics Committee of the Korea University Guro Hospital.

\section{Discussion}

PMPM is an extremely rare tumor accounting for 2-3\% of all cardiac and pericardial tumors (10). The association between asbestos exposure and malignant pleural mesothelioma is well documented, but the etiology of PMPM remains to be elucidated (16). Previous cases have rarely reported obvious exposure to asbestos $(4,17)$. However, in the Lombardy Mesothelioma Registry, taken from a highly industrialized region of northern Italy, 5/7 patients (71.4\%) with PMPM reported occupational asbestos exposure (18). In the present case, the patient had a history of working in soldering in the 1990s. However, it is difficult to connect this history with asbestos, as the patient's work was not associated with construction, automobile repair or asbestos textiles, which are the occupations most highly associated with asbestos exposure.
Owing to its atypical and non-specific symptoms, including dyspnea, coughing or chest pain, physicians are likely to misdiagnose PMPM on initial examination of the patient. Numerous patients with PMPM have presented with pericarditis with pericardial effusion $(6,19,20)$. Furthermore, these patients suffered from recurrent pericardial effusion and were diagnosed with PMPM following repetitive investigation using additional imaging tools such as CT and MRI, as well as tissue biopsy of the pericardium, to determine the cause of the pericardial effusion. In the present case, the patient had a history of massive pericardial effusion due to an unidentified cause 6 years prior to the current study. Compared with other cases, the time interval between the previous pericardial effusion and the current presentation were too long for a diagnosis of recurrent pericardial effusion in the present case, making it difficult to connect with PMPM. However, PMPM should be considered as a cause of unexplained recurrent pericardial effusion, considering previous cases $(6,7,21,22)$.

For the treatment of PMPM, surgical resection is the treatment of choice for localized disease (12). However, numerous cases are locally advanced, with invasion or infiltration of the myocardium, atria, coronary arteries and the conduction system of the heart. In those cases, complete surgical resection is not possible. In addition, distant metastases in the lung or liver are observed in $25-45 \%$ of cases $(13,14)$. Patients with distant metastasis, such as the present case, and patients who are not candidates for surgery should be administered chemotherapy $(11,14)$. Owing to the rarity of PMPM, a standard chemotherapeutic regimen has not yet been established and older chemotherapeutic drugs have demonstrated little benefit in previous years (2). However, the introduction of pemetrexed, a third-generation antifolate drug, demonstrated a survival benefit when combined with cisplatin in patients with pleural mesothelioma (23), leading to longer survival times for patients with PMPM. In previous cases, several patients who were treated with pemetrexed plus carboplatin or pemetrexed plus cisplatin demonstrated positive responses after receiving only two or three cycles of pemetrexed plus platinum $(14,24,25)$. Following 6-8 cycles of treatment, chemotherapy was stopped or consolidation radiotherapy was added in these cases. Positive responses continued for 6-26 months. Doval et al (14) described a patient who remained alive 26 months subsequent to the conclusion of 6 cycles of pemetrexed plus cisplatin. However, other patients exhibited 6-10 months of durable response, followed by an accelerated deterioration subsequent 
to disease progression $(24,25)$. In patients in whom the response to chemotherapy is positive, it is difficult to determine the appropriate duration of chemotherapy. However, considering the aggressiveness and poor prognosis of PMPM, continuation of pemetrexed monotherapy after several cycles of pemetrexed plus cisplatin may be an appropriate treatment strategy if the patient is able to tolerate the treatment. The number of cycles of pemetrexed plus cisplatin treatment required for maximal response and minimal toxicity remains to be elucidated. With pemetrexed plus cisplatin treatment, physicians should be aware of hematological and non-hematological toxicities, for example peripheral neuropathy, as well as response (26). A treatment schedule of pemetrexed maintenance chemotherapy following four cycles of pemetrexed plus cisplatin is actively used for the treatment of lung cancer. The application of a maximum of 44 cycles of pemetrexed for maintenance chemotherapy was reported without significant toxicities in a phase III study in lung cancer patients (27).

In conclusion, pemetrexed maintenance following pemetrexed plus cisplatin combination therapy is an optimal choice for the chemotherapy of inoperable or metastatic PMPM. Physicians should consider the chemotherapy strategy applied within the present study as a priority for the systemic treatment of PMPM, which is typically associated with a poor prognosis. Further investigation is required to determine the appropriate duration of platinum combination therapy, and research on the efficacy of combined therapy with other treatment strategies, such as radiotherapy or surgery, is warranted.

\section{References}

1. De Rosa AF, Cecchin GV, Kujaruk MR, Gayet EG, Grasso LE and Rigou DG: Malignant mesothelioma of the pericardium. Medicina (B Aires) 54: 49-52, 1994 (In Spanish).

2. Thomason R, Schlegel W, Lucca M, Cummings S and Lee S: Primary malignant mesothelioma of the pericardium. Case report and literature review. Tex Heart Inst J 21: 170-174, 1994.

3. Falkenstern-Ge RF, Kimmich M, Bode-Erdmann S, Friedel G, Ott $\mathrm{G}$ and Kohlhäufl $\mathrm{M}$ : Pleural mesothelioma presenting as periumbilical metastasis: The first clinical documentation. Case Rep Oncol Med 2013: 198729, 2013.

4. Nilsson A and Rasmuson T: Primary Pericardial mesothelioma: Report of a patient and literature review. Case Rep Oncol 2: $125-132,2009$

5. Eren NT and Akar AR: Primary pericardial mesothelioma. Curr Treat Options Oncol 3: 369-373, 2002.

6. Makarawate P, Chaosuwannakit N, Chindaprasirt J, Ungarreevittaya $\mathrm{P}$, Chaiwiriyakul S, Wirasorn K, Kuptarnond C and Sawanyawisuth K: Malignant mesothelioma of the pericardium: A report of two different presentations. Case Rep Oncol Med 2013: 356901, 2013.

7. Choi WS, Im MS, Kang JH, Kim YG, Hwang IC, Lee JM, Lee S, Shin HS, Lee SP and Cho GY: Primary malignant pericardial mesothelioma presenting as acute pericarditis. J Cardiovasc Ultrasound 20: 57-59, 2012.

8. Nana A, Vorilhon C, Adjtoutah D, Azarnoush K, Kissel V, Chabin X, Chailloux A, Belhakem A, Tixier V, Ferrier N, et al: Contribution of magnetic resonance imaging in diagnosis of pericardial mesothelioma: A case report. Ann Cardiol Angeiol (Paris) 61: 370-374, 2012 (In French).
9. Ost P, Rottey S, Smeets P, Boterberg T, Stragier B and Goethals I: F-18 fluorodeoxyglucose PET/CT scanning in the diagnostic work-up of a primary pericardial mesothelioma: A case report. J Thorac Imaging 23: 35-38, 2008.

10. Kaul TK, Fields BL and Kahn DR: Primary malignant pericardial mesothelioma: A case report and review. J Cardiovasc Surg (Torino) 35: 261-267, 1994.

11. Maruyama R, Sakai M, Nakamura T, Suemitsu R, Okamoto T, Wataya H, Nishiyama K, Kamei T and Ichinose Y: Triplet chemotherapy for malignant pericardial mesothelioma: A case report. Jpn J Clin Oncol 36: 245-248, 2006.

12. Fujita K, Hata M, Sezai A and Minami K: Three-year survival after surgery for primary malignant pericardial mesothelioma: Report of a case. Surg Today 44: 948-951, 2014.

13. Papi M, Genestreti G, Tassinari D, Lorenzini P, Serra S, Ricci M, Pasquini E, Nicolini M, Pasini G, Tamburini E, et al: Malignant pericardial mesothelioma. Report of two cases, review of the literature and differential diagnosis. Tumori 91: 276-279, 2005.

14. Doval DC, Pande SB, Sharma JB, Rao SA, Prakash N and Vaid AK: Report of a case of pericardial mesothelioma with liver metastases responding well to pemetrexed and platinum-based chemotherapy. J Thorac Oncol 2: 780-781, 2007.

15. Bilchick KC and Wise RA: Paradoxical physical findings described by Kussmaul: Pulsus paradoxus and Kussmaul's sign. Lancet 359: 1940-1942, 2002.

16. Attanoos RL: Asbestos-related lung disease. Surg Pathol Clin 3: 109-127, 2010.

17. Fujiwara H, Kamimori T, Morinaga K, Takeda Y, Kohyama N, Miki Y, Inai K and Yamamoto S: An autopsy case of primary pericardial mesothelioma in arc cutter exposed to asbestos through talc pencils. Ind Health 43: 346-350, 2005.

18. Mensi C, Giacomini S, Sieno C, Consonni D and Riboldi L: Pericardial mesothelioma and asbestos exposure. Int J Hyg Environ Health 214: 276-279, 2011.

19. Kayatta MO, Dineen SP, Sica G, Puskas JD and Pickens A: Primary pericardial mesothelioma in a 19-year-old presenting as pericarditis. Ann Thorac Surg 96: 680-681, 2013.

20. Gong W, Ye X, Shi K and Zhao Q: Primary malignant pericardial mesothelioma - a rare cause of superior vena cava thrombosis and constrictive pericarditis. J Thorac Dis 6: E272-E275, 2014.

21. Molina Garrido MJ, Mora Rufete A, Rodriguez-Lescure A, Cascon Perez JD, Ardoy F, Guillen Ponce C and Carrato Mena A: Recurrent pericardial effusion as initial manifestation of primary diffuse pericardial malignant mesothelioma. Clin Transl Oncol 8: 694-696, 2006.

22. Butz T, Faber L, Langer C, Korfer J, Lindner O, Tannapfel A, Muller KM, Meissner A, Plehn G, Trappe HJ, et al: Primary malignant pericardial mesothelioma - a rare cause of pericardial effusion and consecutive constrictive pericarditis: A case report. J Med Case Rep 3: 9256, 2009.

23. Vogelzang NJ, Rusthoven JJ, Symanowski J, Denham C, Kaukel E, Ruffie P, Gatzemeier U, Boyer M, Emri S, Manegold C, et al: Phase III study of pemetrexed in combination with cisplatin versus cisplatin alone in patients with malignant pleural mesothelioma. J Clin Oncol 21: 2636-2644, 2003.

24. Santos C, Montesinos J, Castañer E, Sole JM and Baga R: Primary pericardial mesothelioma. Lung Cancer 60: 291-293, 2008.

25. Fujimoto N, Gemba K, Wada S, Ono K, Fujii Y, Ozaki S, Ikeda T, Taguchi K, Kunitomo T and Kishimoto T: Malignant pericardial mesothelioma with response to chemotherapy. J Thorac Oncol 4: 1440-1441, 2009.

26. Niyikiza C, Hanauske AR, Rusthoven JJ, Calvert AH, Allen R, Paoletti P and Bunn PA Jr: Pemetrexed safety and dosing strategy. Semin Oncol 29 (Suppl 18): 24-29, 2002.

27. Paz-Ares LGde Marinis FDediu MThomas MPujol JLBidoli PMolinier OSahoo TPLaack EReck M, et al: PARAMOUNT: Final overall survival results of the phase III study of maintenance pemetrexed versus placebo immediately after induction treatment with pemetrexed plus cisplatin for advanced nonsquamous nonsmall-cell lung cancer. J Clin Oncol 31: 2895-2902, 2013. 